\title{
Variabilidade de diagnósticos psicológicos frente à avaliação dermatológica da escoriação psicogênica*
}

\section{Variability of psychological diagnosis in patients with psychogenic excoriation}

\author{
Cinthia Janine Meira Alves ${ }^{1}$ \\ Renata Bilion Ruiz Prado ${ }^{3}$
}

\author{
Antônio Carlos Ceribelli Martelli ${ }^{2}$ \\ Mariane da Silva Fonseca ${ }^{4}$
}

\begin{abstract}
Resumo: Dentre as dermatoses psiquiátricas, a escoriação psicogênica caracteriza-se pela confissão do paciente de provocar as lesões, sem apresentar doença dermatológica de base. Verificou-se que o assunto tem sido pouco abordado na literatura, com enfoque nos diagnósticos psicológicos. Dados epidemiológicos indicaram uma prevalência de 2 a $3 \%$, em relação à população geral, $2 \%$, entre pacientes dermatológicos, e $9 \%$, em pacientes com prurido, com predomínio significativo em mulheres. O artigo relata três casos brasileiros e discute o plano terapêutico constituído de equipe interdisciplinar, permitindo indicações psicoterapêuticas e medicamentosas específicas a cada caso.
\end{abstract}

Palavras-chave: Dermatologia; Diagnóstico; Psicologia; Terapêutica

\begin{abstract}
Among psychiatric dermatoses, the psychogenic excoriation is characterized by patient's confession to provoking the lesions, without presenting any dermatological underlying pathology. This topic has been rarely approached in the literature, focusing on the diversity of psychological diagnoses. Epidemiological data indicated that prevalence varies from 2 to $3 \%$ in the general population, $2 \%$ among dermatological patients and $9 \%$ in patients with itching, with significant prevalence in women. The present article report three Brazilian cases and discusses the therapeutic plan based on multidisciplinary teamwork, including indications of psychotherapy and specific drug therapy to each case.
\end{abstract}

Keywords: Dermatology; Diagnosis; Psychology; Therapeutics

\section{INTRODUÇÃO}

A interface entre as áreas da saúde tem se tornado um campo fértil para investigação de fenômenos complexos. Pela necessidade de agregar conhecimentos pertinentes entre campos diferentes, surgiu a Dermatologia Integrativa, foco da Psiconeuroimunologia, que visa à inter-relação da mente e corpo do indivíduo e o manejo de formas alternativas de minimizar o estresse e otimizar a eficiência do tratamento. ${ }^{1}$
Comprometida com a tarefa de integrar o trabalho dos dermatologistas e psicólogos, a Psicodermatologia tem se proposto à pesquisa e tratamento de dermatoses que apresentam fatores mais susceptíveis a determinantes psicológicos. ${ }^{2,3}$

Torres enfatiza a importância da compreensão dos quadros psiquiátricos, presentes nas dermatoses, assim, a discussão de caso, em equipe interdisciplinar,

Recebido em 12.08.2008.

Aprovado pelo Conselho Editorial e aceito para publicação em 16.10.2008.

* Trabalho realizado no Serviço de Dermatologia do Instituto "Lauro de Souza Lima" (ILSL) - Bauru (SP), Brasil.

Conflito de interesse: Nenhum / Conflict of interest: None

Suporte financeiro: Nenhum / Financial funding: None

Médica Residente em Dermatologia do Instituto "Lauro de Souza Lima" - Bauru (SP), Brasil.

Médico dermatologista Chefe de Seção Técnica de Saúde em Dermatologia do Instituto "Lauro de Souza Lima" - Bauru (SP), Brasil

Mestre em Psicologia - Pesquisadora Científica do Instituto "Lauro de Souza Lima" - Bauru (SP), Brasil.

Mestre em Psicologia - Psicóloga do Instituto "Lauro de Souza Lima" - Bauru (SP), Brasil 
pode se revelar uma estratégia eficaz pela complexidade dos sintomas que o paciente pode apresentar. ${ }^{4,5}$

Martelli ${ }^{6}$ esclarece sobre os transtornos psiquiátricos associados a quadros dermatológicos obscuros, nos quais os exames laboratoriais apresentam resultados negativos. Dentre esses transtornos, a escoriação psicogênica é alvo de objeto de estudo, frente à escassez de pesquisas. Caracteriza-se pela confissão do paciente de provocar as lesões, sendo essa a chave do diagnóstico diferencial com a dermatite factícia. ${ }^{6}$ Esse transtorno apresenta também outras designações. ${ }^{6.8}$

Dados epidemiológicos indicaram uma prevalência de 2 a $3 \%$, em relação à população geral, $2 \%$, entre pacientes dermatológicos, e $9 \%$, em pacientes com prurido. As escoriações ocorrem nos locais mais facilmente alcançados pelas mãos. O predomínio desse transtorno é significativo em mulheres, podendo haver em qualquer idade. ${ }^{6}$

As condições psicopatológicas subjacentes mais comuns são: depressão e ansiedade, transtorno de personalidade borderline, transtorno dismórfico corporal, transtorno de uso de substâncias, transtornos alimentares, tricotilomania e cleptomania. Podem ser identificados também comportamento autodestrutivo, dificuldade de expressar raiva e transtorno obsessivo-compulsivo. ${ }^{6}$

Segundo o Manual Diagnóstico e Estatístico de Transtornos Mentais, a escoriação psicogênica pode ser classificada, dentro do capítulo de Transtornos de Controle de Impulsos, cujo ato de escoriar-se é executado em resposta ao sentimento de tensão aumenta$\mathrm{da}$, ou associado a tentativas de resistir a esse sentimento.'

Atualmente, a conduta terapêutica medicamentosa da escoriação psicogênica consiste na indicação de antidepressivos e antipsicóticos. Dentre as possibilidades terapêuticas não-medicamentosas, a literatura refere à psicoterapia comportamental, focal ou breve. ${ }^{10-12}$

\section{RELATO DOS CASOS}

Caso 1: paciente do sexo feminino, branca, casada, 45 anos, apresentava quadro de escoriação psicogênica, onicofagia e onicotilomania. Os exames laboratoriais apresentaram resultados negativos. Prescreveu-se antidepressivo tricíclico, e psicoterapia. Após 2 anos de acompanhamento, ainda mantinha escoriações e queilofagia (Figura 1), sendo prescritas, também, inibidor seletivo de recaptação de serotonina. A paciente está apresentando apenas queilofagia, estando em uso de inibidor seletivo de recaptação de serotonina e psicoterapia. Constatou-se transtorno de personalidade obsessivo-compulsiva. ${ }^{9}$ A paciente revela preocupação com organização, perfeccionismo e controle sobre os outros; excessiva dedicação ao tra-

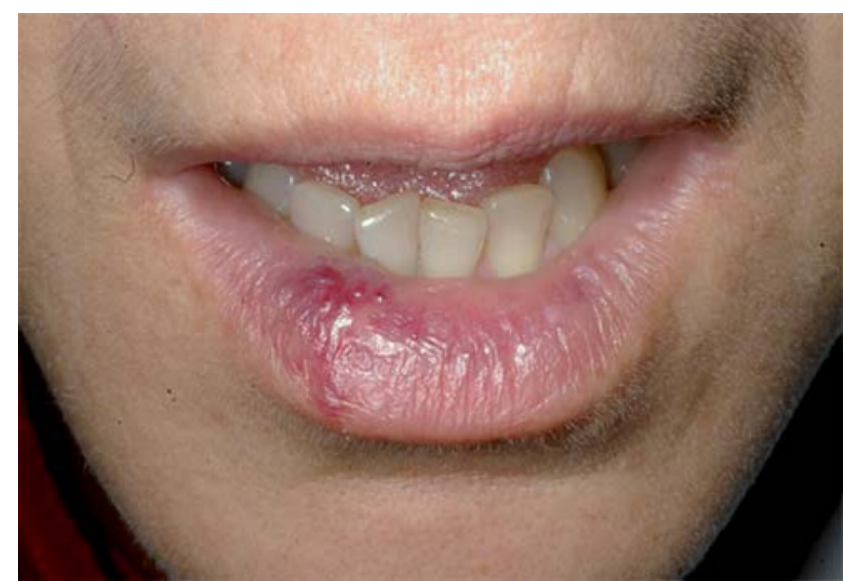

FIGURA 1: Pápulas eritemato-vinhosas agrupadas em lábio inferior devido a mordidas repetitivas no mesmo local (queilofagia)

balho, excluindo as atividades de prazer; demonstra atenção exagerada a regras e listas; segue princípios morais rígidos. Os sentimentos reprimidos são percebidos pela ambivalência entre satisfazer o outro e, ao mesmo tempo, desejá-lo distante. Atualmente, a paciente demonstra autopercepção de seus limites, conclui tarefas domésticas, sem seguir regras, melhora da auto-estima e redução dos sintomas clínicos.

Caso 2: paciente do sexo feminino, 40 anos, branca, casada, reside com dois filhos e o marido, apresenta quadro extenso de escoriações, há oito anos (Figura 2). Relata que o filho e o marido possuíam lesões semelhantes. Os exames laboratoriais resultaram negativos. Durante a infância, a paciente produzia as mesmas lesões nas costas da mãe, princi-

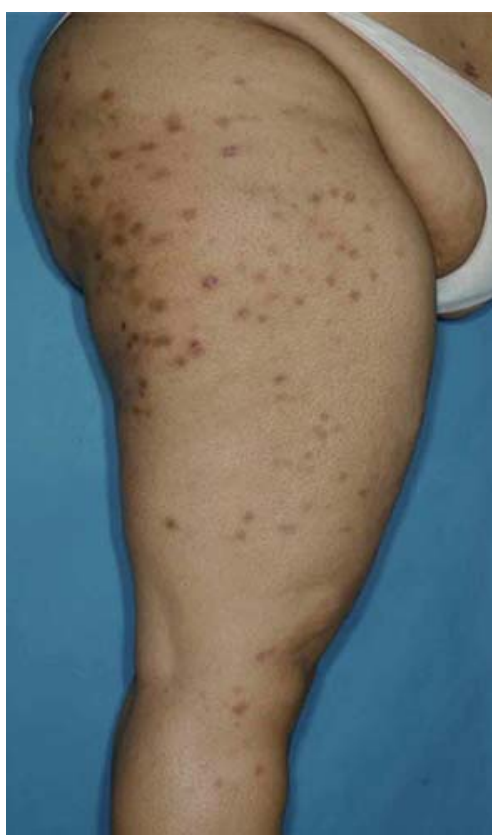

Figura 2: Escoriações em perna, nádega e região inferior do abdome (prurido biopsiante) e algumas lesões residuais hiperpigmentadas em face lateral da coxa 
palmente, no período noturno e registrava compulsividade de gastos e alimentos. Com o diagnóstico de escoriação psicogênica, foram prescritos inibidor seletivo de recaptação de serotonina e antidepressivo tricíclico, encaminhada para psicoterapia. Foi diagnosticado transtorno de personalidade Borderline, devido à apresentação de instabilidade nos relacionamentos interpessoais, auto-imagem, afetos e impulsividade. Atualmente, verifica-se na paciente compreensão da escoriação, como um processo para extravasar conteúdos emocionais reprimidos.

Caso 3: paciente do sexo feminino, 49 anos, solteira, reside com a mãe, apresentava escoriações em membros (Figura 3), alterações peri-ungueais e ungueais dos háluxes (Figura 4), acompanhada de ganho ponderal. Diagnosticou-se escoriação psicogênica e prescrito antidepressivo tricíclico, com controle das lesões. Durante o seguimento, demonstrou-se piora importante, com surgimento de escoriações em face e dorso, associadas a acidente automobilístico e perda do emprego, sendo então acrescentado inibidor seletivo de recaptação de serotonina. Durante o acompanhamento, surgiu episódios de piora, ansiedade, quadro de dermatite de contato nas mãos (teste de contato indicou positivo para thimerosal e sulfato de níquel). O seguimento foi irregular, com resposta insatisfatória às medicações prescritas, assim, introduziu-se antidepressivo atípico. Foi diagnosticado transtorno do controle de impulsos e transtorno alimentar. Detectou-se instabilidade nos relacionamentos interpessoais e impulsividade na adolescência e reconhecido transtorno de personalidade borderline. Atualmente, a paciente apresenta maior senso de controle, melhora na capacidade de auto-expressão e comunicação familiar.

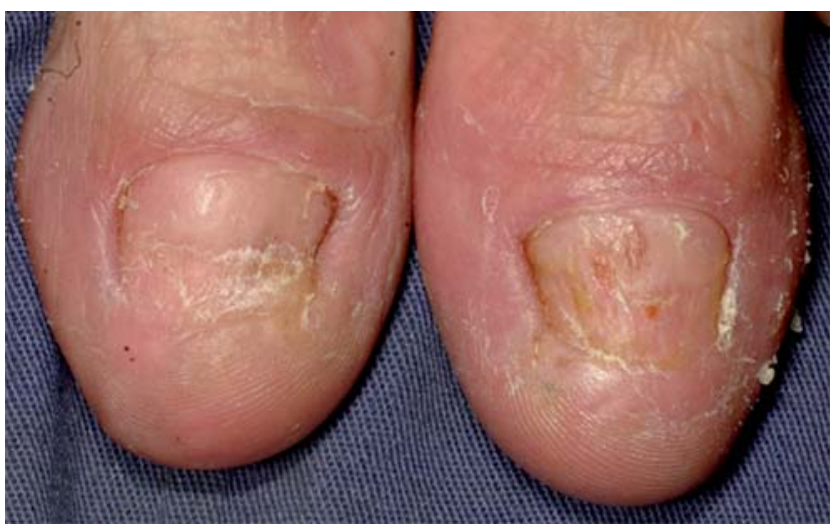

FigURA 3: Onicosquizia, braquioníquia, onicodistrofia do $1 / 3$ distal da lâmina ungueal com depressões transversais e descamação da pele das porções distais dos dedos devido a onicotilomania (lesões produzidas por alicate de cutícula)

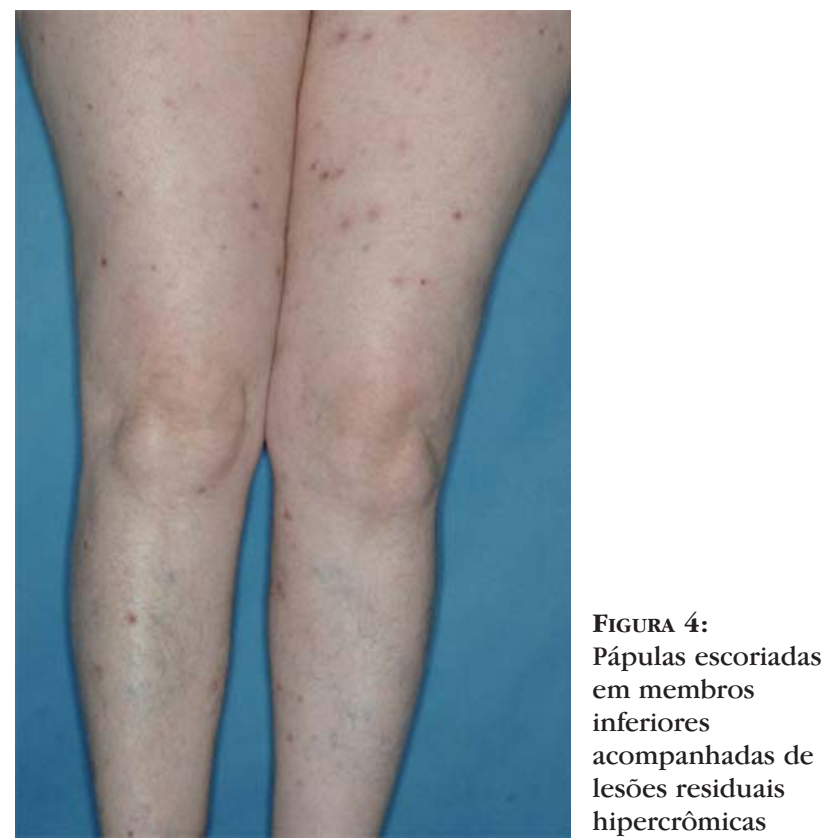

\section{DISCUSSÃO}

$\mathrm{Na}$ análise desses três casos, os sintomas podem ser compreendidos como uma forma do psiquismo manifestar-se simbolicamente. A doença é um modo do organismo reagir a desequilíbrios psicofisiológicos, conduzindo o indivíduo rumo à integração de conteúdos psicológicos, reprimidos, negados ou recalcados. Quando os processos psicológicos encontram barreiras para serem elaborados, por meio da representação verbal, o organismo tende a expressarse em termos somáticos e o conteúdo emocional aparenta um correspondente corporal simbolizador. ${ }^{12}$

Os critérios diagnósticos para escoriação psicogênica foram definidos para os três casos atendidos, porém, por meio de reuniões, em equipe interdisciplinar, detectaram-se aspectos comuns e incomuns nas pacientes.

Embora tenham sido realizados diferentes diagnósticos psicológicos, alguns desses aspectos denotam-se comuns entre os casos: traumas emocionais sofridos durante o desenvolvimento infantil, vividos como abandono; adolescência mal-adaptada, com vínculos afetivos restritos; idade adulta com dificuldades de realizar tarefas que exigem maturidade emocional e habilidade para controlar impulsos.

Outro aspecto convergente, diz respeito à escassez de comprometimento com o tratamento médico, que, inicialmente, limitou a ação da equipe, em relação à eficácia do mesmo. Desta forma, a terapêutica medicamentosa deve considerar as particularidades de cada paciente, sendo que, para sua definição, é imprescindível a realização de estudos de casos clínicos, em equipe interdisciplinar. 
Os pontos incomuns dos casos apresentados referentes ao tipo de transtorno mental foram: transtorno de personalidade borderline; transtorno de personalidade obsessivo-compulsivo; transtorno do controle de impulsos. As variações nos componentes psicológicos envolvem terapêuticas psicológicas, diferentes para cada situação. A utilização da psicoterapia breve, nesses três estudos de casos, permitiu aos profissionais da área a investigação do uso de outras pos- sibilidades terapêuticas. Outro aspecto incomum entre os casos, refere-se à terapêutica medicamentosa que deve considerar as particularidades de cada paciente.

A análise desses três casos representa uma fonte de pesquisa para estudos posteriores, tanto na investigação de diretrizes para avaliação diagnóstica quanto para as alternativas de tratamento.

\section{AGRADECIMENTOS:}

Ao Prof. Dr. Dejair Caitano do Nascimento, Doutor em Farmacologia, Pesquisador Científico do Instituto Lauro de Souza Lima, por ter contribuído com discussões relacionadas aos aspectos farmacológicos das drogas referidas nesse trabalho.

\section{REFERÊNCIAS}

1. Azambuja RD. Dermatologia integrativa: a pele em novo contexto. An Bras Dermatol. 2000;75:393-420.

2. Koo J, Lebwohl A. Psycho dermatology: the mind and skin connection. Am Fam Physician. 2001;64:1873-8.

3. Hoffmann FS, Zogbi H, Fleck P, Müller MC. A integração mente e corpo em psicodermatologia. Psicol Teor Prát. 2005;7:51-60.

4. Torres AR, Smaira SI, Tiosso AM. Distúrbio obsessivocompulsivo e quadros correlatos na clínica dermatológica. An Bras Dermatol. 1995;70:239-43.

5. Wang CK, Lee JY. Monosymptomatic hipochondriacal psychosis complicated by self-inflicted skin ulceration, skull defect and brain abscess. Br J Dermatol. 1997;137:299-302.

6. Martelli AC. Ipele [online]. São José do Rio Preto: Dermatoses psiquiátricas. [update 11 outubro 2006; citado 13 maio 2008]. Disponível em: http:// www.ipele.com.br/livro/capítulo

7. Ferrão YA. Características clínicas do transtorno obsessivo-compulsivo refratário aos tratamentos convencionais [Tese]. São Paulo: Universidade de São Paulo; 2004.

8. Ferrão YA, Ferrão TA, Cunha D. Dermatotilexomania em estudantes de medicina: um estudo piloto. Rev Bras Psiquiatr. 1999;21:109-13.

9. Jorge MR. Manual diagnóstico e estatístico de transtornos mentais DSM-IV. Tradução Dayse Batista. 4 ed. Porto Alegre: Artmed; 2002.
10. Prazeres AM, Souza WF, Fontenelle LF. Terapias de base cognitivo-comportamental do transtorno obsessivocompulsivo: revisão sistemática da última década. Rev Bras Psiquiatr. 2007;29:1-9.

11. March JS, Franklin ME, Leonard H, Garcia A, Moore P, Freeman J, et al. Cognitive- behavior therapy, sertraline, and their combination for children and adolescentes with obsessive-compulsive disorder. Biol Psychiatry. 2007;61:344-7.

12. Muller MC, Ramos DG. Psicodermatologia: uma interface entre psicologia e dermatologia. Psicol Ciênc Prof. 2004;24:76-81.

ENDEREÇO PARA CORRESPONDÊNCIA / MAILING ADDRESS:

Cinthia Janine Meira Alves

Endereço completo: Rodovia Comandante João

Ribeiro de Barros, Km 225/226

17034971 Bauru, SP

Tel./Fax: 14 3103-5887 / 8122-7372 /3103-5914

Email: cinthiameira@yaboo.com.br

Como citar este artigo/How to cite this article: Alves CJM, Martelli ACC, Prado RBR, Fonseca MS . Variabilidade de diagnósticos psicológicos frente à avaliação dermatológica da escoriação psicogênica. An Bras Dermatol. 2009;84(5):534-7. 